\section{Borrelia miyamotoi in Human-Biting Ticks, United States, 2013-2019}

\author{
Guang Xu, Chu-Yuan Luo, Fumiko Ribbe, Patrick \\ Pearson, Michel Ledizet, Stephen M. Rich
}

Author affiliations: University of Massachusetts-Amherst, Amherst, Massachusetts USA (G. Xu, C.-Y. Luo, F. Ribbe, P. Pearson, S.M. Rich); L2 Diagnostics, New Haven, Connecticut, USA (M. Ledizet)

During 2013-2019, Borrelia miyamotoi infection was detected in 19 US states. Infection rate was $0.5 \%-3.2 \%$; of B. miyamotoi-positive ticks, $59.09 \%$ had concurrent infections. B. miyamotoi is homogeneous with 1 genotype from Ixodes scapularis ticks in northeastern and midwestern states and 1 from $I$. pacificus in western states.

DOI: https://doi.org/10.3201/eid2712.204646

$B$ orrelia miyamotoi, a relapsing fever group spirochete (1), was first isolated from Ixodes persulcatus ticks in Japan in 1995 (2) and later detected in Ixodes ticks in the United States and Europe (3-5). Although $B$. miyamotoi bacteria have been mainly detected in $I$. ricinus species complex ticks that transmit $B$. burgdorferi worldwide, the vector specificity needs further study because investigators have found B. miyamotoi in multiple tick species (6). B. miyamotoi has 3 geographically distinct genotypes: Asian, European, and American. In the United States, B. miyamotoi bacteria have been found in field-collected I. scapularis ticks in the northeastern and northern midwestern regions, where the average infection rate is $1.9 \%$ (7). However, an expanded geographic study of the prevalence of
B. miyamotoi in human-biting ticks, its genotypes, and concurrent infections with other tickborne pathogens is warranted.

Human-biting ticks were submitted to the public tick testing program at the University of Massachusetts (Amherst, Massachusetts, USA) during May 2013-December 2019. We extracted DNA from individual ticks using the Epicenter Master Complete DNA and RNA Purification Kits (Lucigen, https:/ / www.lucigen.com). We performed a species-specific quantitative PCR (qPCR) for differentiation of $I$. scapularis and I. pacificus ticks (8). To detect Borrelia bacteria, we first applied a genus-specific detection assay, followed by specific qPCR assays for B. burgdorferi sensu lato and B. miyamotoi. We detected the tickborne pathogens Anaplasma phagocytophilum, Babesia microti, B. mayonii, and Ehrlichia muris-like agent (EMLA) by a multiplex qPCR assay targeting different genes. We used a qPCR assay targeting tick 16S mtDNA gene as an internal control (8). We sequenced 3 partial gene fragments, $16 \mathrm{~S}$ rDNA (16S) (9), flagellin (fla) (6), and glycerophosphodiester phosphodiesterase $(g l p Q)(6)$, for B. miyamotoi samples that were positive by qPCR.

We received and tested 39,198 ticks found on humans for B. miyamotoi during May 2013-December 2019. Of those, $38,855(99.12 \%)$ ticks originated from the continental United States, comprising 18 tick species (Table). Although Ixodes ticks are the main vectors for B. miyamotoi, we did not detect B. miyamotoi DNA in I. affinis, I. angustus, I. cookei, I. dentatus, I. marxi. I. muris, or I. spinipalpis ticks. We detected B. miyamotoi in I. pacificus $(14 / 1,497,0.94 \%)$ and I. scapularis (594/34,621, 1.72\%) ticks.

B. miyamotoi was found in 19 states; infection rates were $0.5 \%-3.2 \%$ (Figure). In the western

\begin{tabular}{lccc}
\hline Table. Human-biting tick species positive for Borrelia miyamotoi and B. burgdorferi sensu lato, United States, 2013-2019 \\
\hline Tick species & Total no. tested & No. B. miyamotoi positive & No. B. burgdorferi s.I. positive \\
\hline Amblyomma americanum & 1,167 & 0 & 0 \\
A. cajennense & 1 & 0 & 0 \\
A. maculatum & 8 & 0 & 0 \\
Dermacentor andersoni & 60 & 0 & 0 \\
D. occidentalis & 91 & 0 & 0 \\
D. variabilis & 1,060 & 0 & 0 \\
Haemaphysalis leporispalustris & 2 & 0 & 0 \\
H. Iongicornis & 7 & 0 & 0 \\
Ixodes affinis & 2 & 0 & 0 \\
I. angustus & 55 & 0 & 0 \\
I. cookei & 123 & 0 & 0 \\
I. dentatus & 48 & 0 & 7 \\
I. marxi & 26 & 0 & 0 \\
I. muris & 9 & 0 & 2 \\
I. pacificus & 1,497 & 14 & 25 \\
I. scapularis & 34,621 & 0 & 1,287 \\
I. spinipalpis & 63 & 0 & 3 \\
Rhipicephalus sanguineus & 15 & 608 & 11.324
\end{tabular}




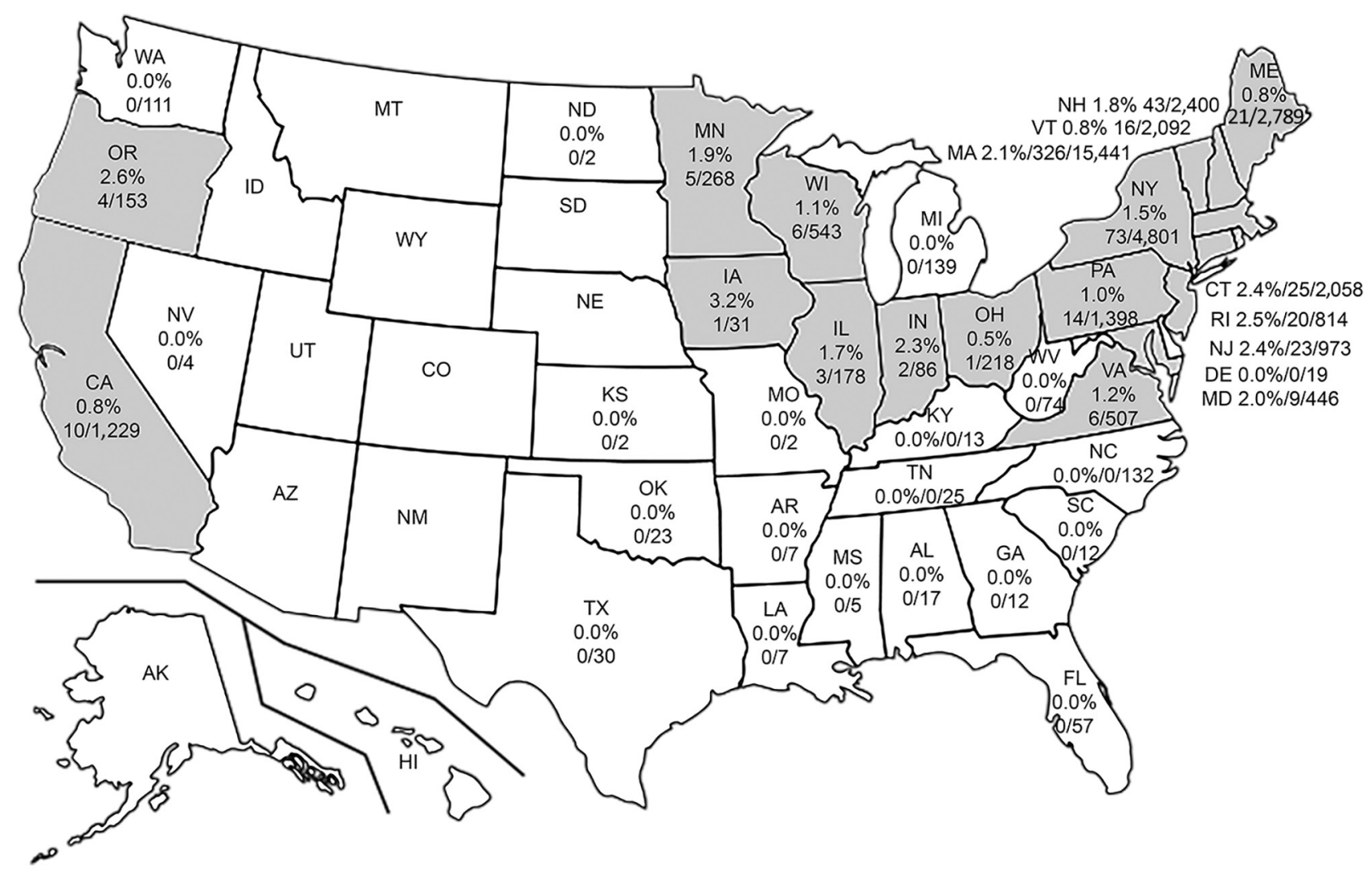

Figure. Borrelia miyamotoi positivity rates in human-biting Ixodes scapularis and I. pacificus ticks, United States, 2013-2019. Gray shading indicates states in which B.miyamotoi was detected in human-biting ticks.

United States, B. miyamotoi was found in I. pacificus ticks in Oregon and California (14/1,497, 0.94\%). Although I. scapularis ticks are distributed across the eastern United States, no B. miyamotoi-positive ticks were detected south of Virginia. B. miyamotoi-positive ticks were concentrated in the Northeast and upper Midwest (594 of 34,621, 1.72\%) (Figure). Lyme disease remains the principal public health concern; the causative agent, $B$. burgdorferi $(11,287 / 34,621$; $32.60 \%, 95 \%$ CI $32.1 \%-33.1 \%)$, was 19 times more prevalent than B. miyamotoi $(594 / 34,621,1.72 \%)$ in $I$. scapularis ticks.

On average, prevalence of $B$. miyamotoi infection in I. scapularis ticks $(1.72 \%, 95 \%$ CI $1.58 \%-1.86 \%)$ was higher than in I. pacificus ticks $(0.94 \%, 95 \%$ CI $0.51 \%-$ $1.56 \%)$. The prevalence of B. miyamotoi in I. pacificus ticks was $1.00 \%(95 \%$ CI $0.53 \%-1.7 \%)$ in adults $(13 / 1,300), 0.53 \%(95 \%$ CI $0.01 \%-2.9 \%)$ in nymphs $(1 / 190)$, and $0.00 \%$ (95\% CI $0 \%-40.1 \%)$ in larvae (0/7). The prevalence of B. miyamotoi in I. scapularis ticks was $1.80 \%(95 \%$ CI $1.64 \%-1.97 \%)$ in adults (456/25,376), $1.54 \%$ (95\% CI 1.29-1.83\%) in nymphs $(133 / 8,615)$, and $0.79 \%$ (95\% CI $0.26 \%-1.84 \%)$ in larvae $(5 / 630)$.
Of 594 B. miyamotoi-positive I. scapularis ticks, $351(59.09 \%)$ had concurrent infections. We found 293 $(49.33 \%)$ I. scapularis ticks had a dual infection with B. miyamotoi: $220(37.04 \%)$ were also infected with $B$. burgdorferi s.l., 43 (7.24\%) with A. phagocytophilum, and $30(5.05 \%)$ with B. microti. We further found 52 $(8.75 \%)$ had a triple infection with B. miyamotoi: 23 $(3.87 \%)$ were also infected with B. burgdorferi s.l. and A. phagocytophilum, $22(3.70 \%)$ with B. burgdorferi s.l. and B. microti, and $7(1.18 \%)$ with $A$. phagocytophilum and B. microti. Six (1.01\%) of the B. miyamotoi-positive ticks had a quadruple infection with $B$. miyamotoi, $B$. burgdorferi s.l., A. phagocytophilum, and B. microti. No ticks with $B$. mayonii or EMLA were additionally infected with B. miyamotoi.

Multilocus sequence typing of the $16 S$, fla, and $g l p Q$ genes revealed 2 distinct $B$. miyamotoi genotypes separated by their tick vectors, I. scapularis ticks in the Northeast and upper Midwest and I. pacificus ticks in the West (Appendix, https://wwwnc.cdc.gov/EID/ article/27/12/20-4646-App1.pdf). Whereas the $16 \mathrm{~S}$ gene sequences were identical among all isolates, variable sites were found among fla and $g l p Q$ nucleotide sequences. Among 14 I. pacificus tick-borne 
B. miyamotoi isolates, all fla and $g l p Q$ sequences were identical. A previously reported A/G substitution in B. miyamotoi fla sequences from I. pacificus ticks $(5,9)$ was outside of our sequenced fla fragment (Appendix). The genetic identity between the 2 tick speciesspecific genotypes was 0.996 for fla and 0.986 for $g l p Q$. Unlike heterogeneous $B$. burgdorferi populations, $B$. miyamotoi appears to be very homogeneous within its respective tick vectors.

\section{About the Author}

$\mathrm{Dr}$. $\mathrm{Xu}$ is a research professor in the department of microbiology, University of Massachusetts-Amherst. His research interests include ticks and tickborne diseases.

\section{References}

1. Krause PJ, Fish D, Narasimhan S, Barbour AG. Borrelia miyamotoi infection in nature and in humans. Clin Microbiol Infect. 2015;21:631-9. https:// doi.org/10.1016/ j.cmi.2015.02.006

2. Fukunaga M, Takahashi $Y$, Tsuruta $Y$, Matsushita O, Ralph D, McClelland M, et al. Genetic and phenotypic analysis of Borrelia miyamotoi sp. nov., isolated from the ixodid tick Ixodes persulcatus, the vector for Lyme disease in Japan. Int J Syst Bacteriol. 1995;45:804-10. https:/ / doi.org/ 10.1099/00207713-45-4-804

3. Scoles GA, Papero M, Beati L, Fish D. A relapsing fever group spirochete transmitted by Ixodes scapularis ticks. Vector Borne Zoonotic Dis. 2001;1:21-34. https:/ / doi.org/ 10.1089/153036601750137624

4. Bunikis J, Tsao J, Garpmo U, Berglund J, Fish D, Barbour AG. Typing of Borrelia relapsing fever group strains. Emerg Infect Dis. 2004;10:1661-4. https:// doi.org/10.3201/eid1009.040236

5. Mun J, Eisen RJ, Eisen L, Lane RS. Detection of a Borrelia miyamotoi sensu lato relapsing-fever group spirochete from Ixodes pacificus in California. J Med Entomol. 2006;43:120-3. https:/ / doi.org/10.1093/jmedent/43.1.120

6. Jiang BG, Jia N, Jiang JF, Zheng YC, Chu YL, Jiang RR, et al. Borrelia miyamotoi infections in humans and ticks, northeastern China. Emerg Infect Dis. 2018;24:236-41. https://doi.org/10.3201/eid2402.160378

7. Barbour AG, Bunikis J, Travinsky B, Hoen AG, Diuk-Wasser MA, Fish D, et al. Niche partitioning of Borrelia burgdorferi and Borrelia miyamotoi in the same tick vector and mammalian reservoir species. Am J Trop Med Hyg. 2009;81:1120-31. https://doi.org/10.4269/ ajtmh.2009.09-0208

8. Xu G, Pearson P, Dykstra E, Andrews ES, Rich SM. Human-biting Ixodes ticks and pathogen prevalence from California, Oregon, and Washington. Vector Borne Zoonotic Dis. 2019;19:106-14. https://doi.org/10.1089/vbz.2018.2323

9. Cook VJ, Fedorova N, Macdonald WP, Lane RS, Barbour AG. Unique strain of Borrelia miyamotoi in Ixodes pacificus ticks, California, USA. Emerg Infect Dis. 2016;22:2205-7. https:/ / doi.org/10.3201/eid2212.152046

Address for correspondence: Guang $\mathrm{Xu}$, University of Massachusetts - Microbiology, Fernald Hall Room B1, 270

Stockbridge Rd, University of Massachusetts, Amherst, MA 01003, USA; email: gxu@umass.edu

\section{Wohlfahrtiimonas chitiniclastica Monomicrobial Bacteremia in a Homeless Man}

\author{
Omar Harfouch, Paul M. Luethy, Mandee Noval, \\ Jonathan D. Baghdadi
}

Author affiliation: University of Maryland Medical Center, Baltimore, Maryland, USA

DOI: https://doi.org/10.3201/eid2712.210327

We report a case of septic shock attributable to monomicrobial bloodstream infection secondary to Wohlfahrtiimonas chitiniclastica infection. This case suggests that $W$. chitiniclastica likely possesses the virulence to cause severe disease. Culture-independent techniques were essential in the identification of this organism, which enabled selection of appropriate therapy.

Tn August 2020, a 63-year-old homeless man with a Lhistory of deep vein thrombosis and chronic venous insufficiency was found in his truck, unconscious and covered in feces and maggots. He reportedly had been parked in a single parking spot in rural Maryland, USA, for 3 days. His blood pressure in the field was too low to be quantified, and he was admitted to a community hospital in septic shock. Blood cultures were drawn before establishing intravenous access for administration of vancomycin, piperacillin/ tazobactam, and crystalloid. After being stabilized, he was transferred to our hospital, a tertiary care center in Baltimore, Maryland, USA, where surgeons performed superficial surgical debridement of his lower extremities and removed maggots by using a scrub brush with the patient under anesthesia in the operating room. We discarded the maggots, and they were not submitted for identification.

The patient's leukocyte count on arrival was $38.6 \mathrm{~K} / \mu \mathrm{L}$ (reference range $4.5-11.0 \mathrm{~K} / \mu \mathrm{L}$ ), his creatinine $6.86 \mathrm{mg} / \mathrm{dL}$ (reference range $0.7-1.5 \mathrm{mg} /$ $\mathrm{dL}$ ), and his lactic acid $3.5 \mathrm{mmol} / \mathrm{L}$ (reference range 0.5-2.2 mmol/L). He had elevated transaminases, an aspartate aminotransferase level of $436 \mathrm{U} / \mathrm{L}$ (reference range 17-59 $\mathrm{U} / \mathrm{L}$ ) and alanine transaminase of $174 \mathrm{U} / \mathrm{L}$ (reference range 0-49 U/L). A computed tomography scan of the lower extremities showed ulceration of the anterior right lower leg with edema and fat stranding of the subcutaneous tissue without fluid collection or gas. A magnetic resonance imaging of his left foot showed no evidence of osteomyelitis.

On day 2 of hospitalization, transient hemodynamic instability necessitated initiation of 